\title{
NUMERICAL EXPERIMENTS ON TURBULENT FLOW USING THE RANDOM VORTEX METHOD
}

\author{
JONATHAN LAITONE* \\ Department of Mechanical Engineering and Applied Mechanics, The University of Michigan, Ann Arbor, Michigan, U.S.A.
}

\begin{abstract}
SUMMARY
Chorin's random vortex method is used to predict the growth of a large-scale coherent vortex structure in the early stages of the development of turbulence in a two-dimensional co-flowing shear layer. The numerical algorithm has been simplified to such an extent that the numerical analysis can be performed on a microcomputer. The numerical solution exhibits the same early turbulent instabilities and vorticity pairings as found in recent flow-visualization experiments. In addition the results are in reasonable agreement with experimental measurements of mean velocity, root mean square fluctuations and Reynolds stresses. One could thus test the shear layer sensitivity to initial conditions and the upsteam boundary conditions.
\end{abstract}

\section{INTRODUCTION}

In a co-flowing shear layer there exists a region of flow conditions where the turbulent structure exhibits a distinct two-dimensional character. ${ }^{1,2}$ This occurs primarily in the formative stages of an early turbulent regime, beyond which the flow structure becomes affected by three-dimensional phenomena.

Recent advances in numerical vortex methods due to Chorin ${ }^{3-5}$ made them particularly suitable for the analysis of the mechanism of large-scale vortex structures in turbulent flows. The two-dimensionality of early turbulence allows the vorticity to be treated as a scalar quantity. In this way a numerical model can be constructed which, although neglecting vortex stretching and tilting, is capable of accurately predicting the scalar vorticity transport. Unlike finite difference methods, the vortex method of Chorin is capable of resolving multiple length scales, and is devoid of numerical instabilities at high Reynolds numbers. Furthermore, it maintains computational efficiency by partitioning small regions of high fluid shear (of particular physical interest) into arbitrary small computational elements.

These small regions interact on a large-scale to reveal coherent large-scale structures of the flow field. Although three-dimensional vortex codes have been applied to turbulence, ${ }^{6}$ their

\footnotetext{
* Jonathan Laitone was an Assistant Professor in Mechanical Engincering at the University of Michigan when he was killed, along with ten other young men, on 21 June 1981, in the worst mountain climbing accident in U.S. history. This occurred on Mt. Rainier near Seattle, Washington, when a huge portion of a glacier fell and was shattered into thousands of ice blocks. Among the eleven men who were killed instantly were two acronautical engineers from England. Eighteen of the original climbing group miraculously survived.

This manuscript was recently found when Jonathan's father was clearing out his son's files. Then Drs. Tzong-Hsi Chen and Michael Hooven, who as graduate students had worked with Jonathan on this project, were able to locate the computer print-outs. Finally, the manuscript was edited by Professor Antoni Oppenheim and Alexandre Chorin at the University of California, Berkeley, and Professor Ahmed Ghoniem at the Massachusetts Institute of Technology.
} 
deteriorating accuracy at high Reynolds numbers, when the numerical diffusivity exceeds the physical viscous effects, makes their use as a research tool of questionable value.

Early investigations by Rosenhead ${ }^{7}$ showed that vortex methods can produce the same coalescence of vorticity as observed experimentally. Other investigators, ${ }^{8,9}$ using more accurate numerical procedures, produced similar results for complex flows. Although the calculations required relatively long computing time, they demonstrated that the vortex method can predict the correct shear layer growth rate, eddy pairing, turbulent shear stress profile and root mean square velocity fluctuations measured at moderate Reynolds numbers.

Here it is shown that early turbulence exhibits inertial and viscous characteristics which allow considerable simplifications of the numerical algorithm. Besides providing an accurate prediction of 'early turbulence', the algorithm is sufficiently economical for use on a microcomputer, lending itself as a relatively simple technique for numerical experiments.

\section{METHOD}

In the vortex method, computational particles of vorticity, or "blobs', are tracked in the Lagrangian sense. This is accomplished as follows.

According to Kelvin's theorem the circulation in a material blob of fluid is time-invariant. Hence we can partition the vorticity $\xi$ in a fluid domain into non-overlapping blobs and assign the circulation of that region to a single point $x_{i}$, yielding

$$
\xi=\sum_{j=1}^{N} \xi_{j}
$$

This sum converges in the sense of distributions to the total vorticity as the blob size becomes vanishingly small.

By the method of fractional steps ${ }^{10}$ we solve first for advection and then for diffusion. The Navier-Stokes equations are for this purpose expressed in terms of the vortex transport equation

$$
\frac{\mathrm{D} \xi}{\mathrm{D} t}=v \nabla^{2} \xi
$$

subject to the continuity requirement

$$
\operatorname{div} \mathbf{u}=0
$$

and initial and boundary conditions: $\mathbf{u}(-\infty, t)=1 ; u(t)=0$.

$$
\xi(x, t=0)=\xi(0)
$$

\section{Solution for $R \rightarrow \infty$}

The vorticity, $\xi$, for each blob is governed by the Poisson equation for the stream function $\psi_{j}$

whence

$$
\nabla^{2} \psi_{j}=-\xi_{j}
$$

$$
u_{j}=\frac{\partial \psi_{j}}{\partial y}, \quad v_{j}=-\frac{\partial \psi_{j}}{\partial x}
$$

Its solution is expressed in terms of the convolution of the Green function

$$
\psi_{j}=G_{\mathrm{a}}^{*} \xi_{j}
$$


where $G_{\mathbf{a}}$ is smoothed near the origin to remove the singularity. ${ }^{3}$

The motion of a blob at $x_{i}$ is then determined by integrating

$$
\begin{aligned}
& \frac{\mathrm{d} x_{i}}{\mathrm{~d} t}=u_{i}=\sum_{j \neq i}^{N} \frac{\partial}{\partial y} \psi_{j}, \quad i=1, \ldots, N \\
& \frac{\mathrm{d} y_{i}}{\mathrm{~d} t}=v_{i}=-\sum_{j \neq 1}^{N} \frac{\partial}{\partial x}-\psi_{j}, \quad i=1, \ldots, N
\end{aligned}
$$

Hald ${ }^{11}$ proved that this approximate solution converges to that of the Euler equation with an order of accuracy that depends on the form of the smoothing function.

\section{Solution for $R \neq \infty$}

In this case, the diffusion of vorticity is manifested by a random walk, as shown by Chorin. ${ }^{3}$ The walk displaces each blob by a distance sampled from a Gaussian random variable of zero mean, and its variance is given by

$$
\frac{\sigma^{2}}{L^{2}}=\frac{2 \Delta t}{R} \frac{\Delta U}{L}
$$

This approximates the Green function expressing the solution of the vorticity diffusion, namely

$$
G(x, t)=\frac{1}{\sqrt{(4 \pi t \Delta U L / R)}} \exp \left\{-\frac{x^{2} R / L}{4 t \Delta U}\right\}
$$

Since the standard deviation of a random walk is a measure of the expected statistical error, it follows from (7) that, in contrast to a finite difference algorithm, errors get smaller when the Reynolds number, $R$, increases.

Errors are also introduced in the numerical initial conditions (3) when the continuous and essentially smooth vorticity field is discretized numerically into pointwise particles of vorticity or blobs. These errors can have a significant influence on the accuracy of the method. Controlling the errors, and identifying the sources which contribute to them is therefore of crucial importance for successful application of the random vortex method to a practical flow problem, as illustrated, for example, by Laitone ${ }^{12}$ in the case of gas flow with solid particles.

\section{EXPERIMENT}

The subject of the numerical experiments is the classical case of the formation of a turbulent shear layer in a planar flow behind a splitter plate, as described in particular by Roshko ${ }^{13}$ and explored experimentally by Brown and Roshko, ${ }^{1}$ among others. The vortex vector field we computed, corresponding to the wind-tunnel experiments of Batt ${ }^{14}$ where the velocity above the plate was $U_{1}=700 \mathrm{~cm} / \mathrm{s}$ and that below the plate was $U_{2}=70 \mathrm{~cm} / \mathrm{s}$, is presented in Figure 1 .

Vorticity was generated at the rear edge of the plate to satisfy the Kutta condition. The circulation strength of the vortex sheet satisfying this condition is $\Gamma=(\Delta U) L$, where $L$ is a characteristic length scale. For numerical analysis the sheet is modelled by $N$ discrete vortices per unit length, each over a segment of length $h=L / N$. To satisfy the Courant stability condition, the computational time step, $\Delta t$, is such that vortices are advected a distance $h=U \Delta t$. The circulation of each discrete vortex is thus $\Gamma_{j}=(\Delta U) U \Delta t$.

Physically the vortex sheet satisfies the time-mean vorticity distribution in the flow field. 


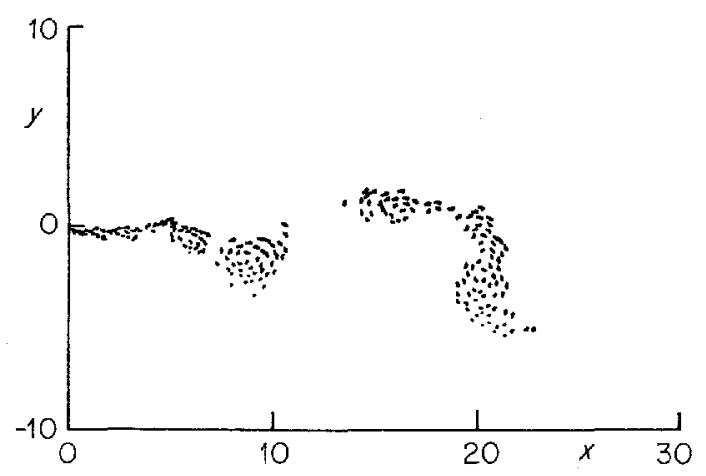

Figure 1. Flow simulation computed by the discrete vortex method. The splitter plate ends at $x=0$ with $\Delta U \Rightarrow 630 \mathrm{~cm} / \mathrm{s}$; $R=4240 ; \xi=-0 \cdot 1250 ; t=20 \cdot 45 \mathrm{~s}$

Mathematically this forces the initial flow to conform to its elliptic nature, rather than developing through a parabolic marching scheme.

The resulting flow pattern depicted in Figure 1 exhibits the organized vorticity coalescence observed experimentally. This calculation was performed on a desk-top Hewlett- Packard microcomputer in $48 \mathrm{~h}$.

\section{SHEAR LAYER}

Numerical simulations duplicated the air flow experiments of Batt, ${ }^{14}$ where $U_{1}=700 \mathrm{~cm} / \mathrm{s}$ and $U_{2}=70 \mathrm{~cm} / \mathrm{s}$. Ashurst ${ }^{15}$ has shown that the random walk produces the $\sqrt{ } x$ momentum thickness growth rate, with a transition to linear growth in the turbulent region. Neglecting the random walk still yielded the correct linear growth rate in the downstream turbulent region. However, his calculations failed to predict the early growth rate. Ashurst concluded that the turbulent growth rate is a consequence of Euler's equations (i.e. no random walk simulation of viscous dissipation).

In the present work we find similar results; this observation also yields an economical modification of the numerical algorithm. In the early stages of the flow the random displacement is introduced in (6a) and (6b); however, it is eliminated after each vortex has moved through the transition point.

The random walk introduces an initial disturbance into the field. Numerically this procedure is in keeping with linear instability theory, where early turbulence is assumed to develop from a two-dimensional instability. Physically this instability is created by the high viscous forces which dominate the inertial forces at the edge of the splitter plate. Here the Reynolds number is very low:

$$
\frac{\text { inertial force }}{\text { viscous force }}=\frac{\rho U^{2} \delta}{\mu U} \ll 1
$$

since the shear layer thickness, $\delta$, is small. As we move downstream the inertial forces tend to dominate over the vorticity diffusion expressed by the random walk. Therefore, this physical process was neglected, on the presumption that downstream the flow is governed by Euler's equations.

The random walk also generates a 'white noise' spectrum; hence the wavelengths of the disturbance is set by the most unstable wave in the initial growth region. This corresponds to 
those vortices most unstable to small perturbations in their position, which in general are those which 'walked' the furthest distance in one time step. The furthest distance a vortex is likely to 'walk' is two standard deviations; this is apparent from (7), from which it follows that

$$
\frac{2 \sigma}{L}=\sqrt{\left(\frac{8 \Delta t \Delta U}{R L}\right)}
$$

Although this relationship is realized only within the framework of numerical modelling, it does present an heuristic picture of the dependence of the transitional nature of the shear layer on the Reynolds number and velocity difference.

Experimental studies by Bradshaw, ${ }^{16}$ as well as others, support the connection between initial disturbance conditions and shear layer structure. For example, Winant and Browand ${ }^{2}$ have shown that periodically forcing a shear flow with controlled disturbances at a fixed frequency can delay the transition point. Acton ${ }^{8}$ also found in a numerical simulation that altering the initial instability by decreasing its amplitude causes a delay in the initial roll-up of the shear layer.

\section{COHERENT STRUCTURE}

Past the region of unstable waves that form by perturbing the separating layer, we find the formation of discrete coherent clusters of vortex blobs, as displayed in Figure 2, produced as a result of the non-linear interaction that occurs in the course of vortex pairing. These interactions are governed by the Biot-Savart law. Further downstream, the clusters roll around each other. This type of vortex pairing, as extensively discussed by Winant and Browand, ${ }^{2}$ is responsible for entraining the irrotational fluid between merging vortex clusters.

In numerical experiments we obtained pairing and triplet mergers of clusters. The type of amalgamation or merging depends highly on small variations in the original vortex structure. Often some vortices 'drop out' of the merger and are left between clusters, as seen in Figure 2. Furthermore, the initial mergers are intermittent.

Physical phenomena of multiple length scales are, in effect, modelled by the random vortex method. Brown and Roshko ${ }^{1}$ suggested that each discrete scale of the coherent structure must participate in an amalgamation event to produce the linear growth rate observed globally. They pointed out that these amalgamation events should exhibit a 'jitter', which is indeed well manifested by numerical results. In order to emphasize this effect we assigned a variable wave number to each discrete vortex. Effectively this produced a long-range cut-off circle, past which the stream function for a vortex blob vanished. In the computer program, the wave number assigned to each discrete vortex was allowed to vary linearly with time. Aref and Siggia ${ }^{9}$ showed

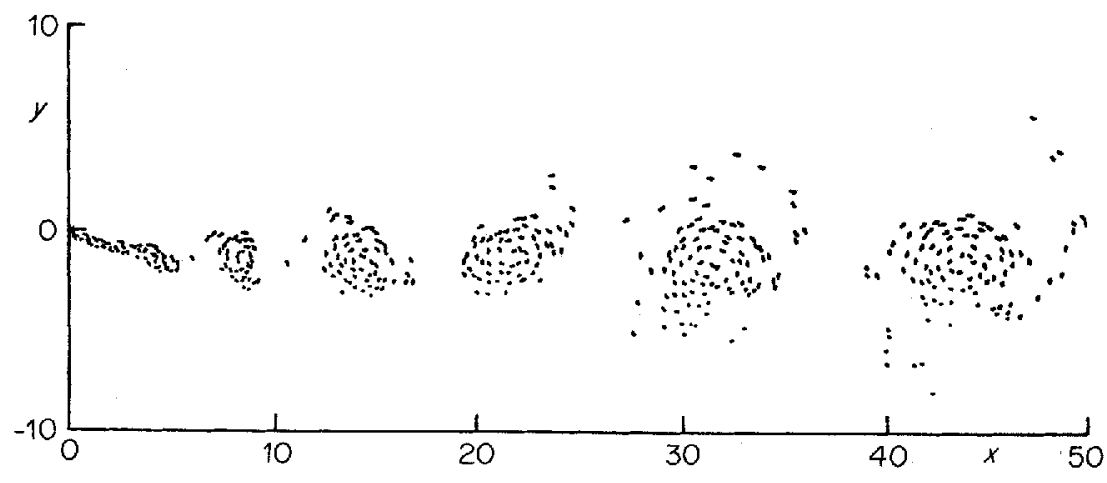

Figure 2. Continuation of Figure 1 with $t=60.95 \mathrm{~s}$ 


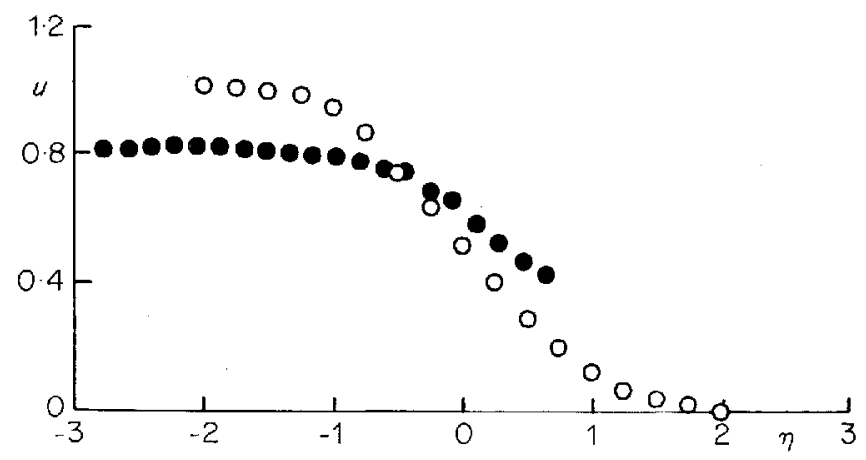

Figure 3. Computed mean horizontal velocity $(-)$, compared with Batt's ${ }^{14}$ data $(O)$

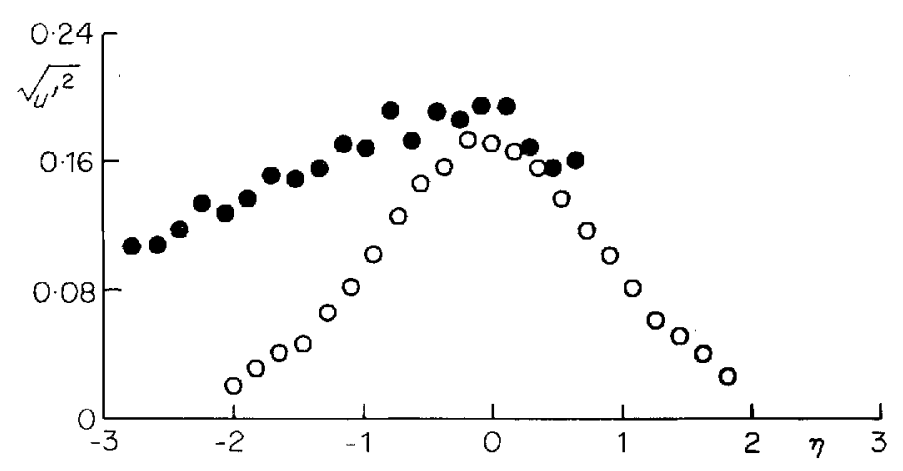

Figure 4. Computed RMS fluctuation in horizontal velocity ( $)$, compared with Batt's ${ }^{14}$ data (O)

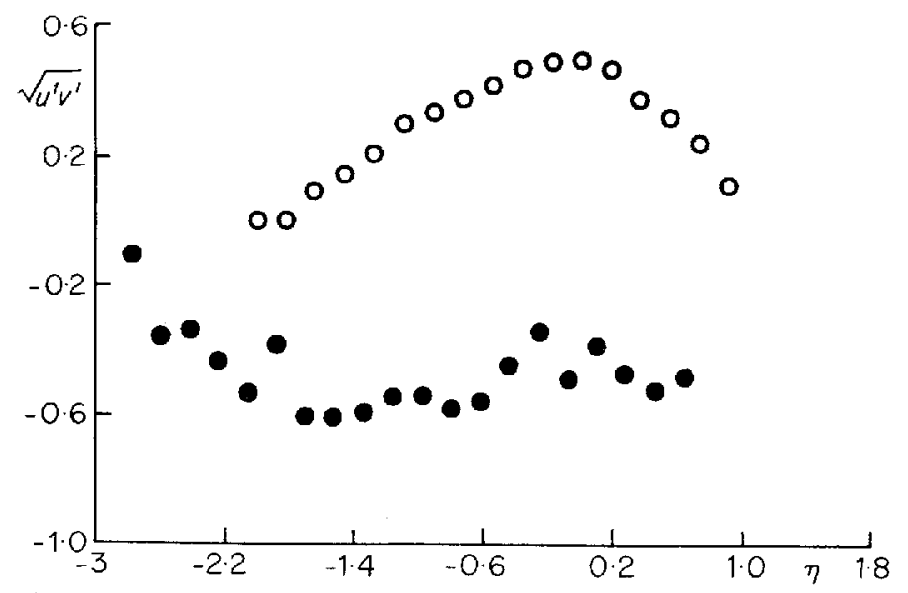

Figure 5. Computed Reynold's stress ( $)$ ), compared with Batt's ${ }^{14}$ data (O) 
that assigning a truncated wavelengths to each discrete vortex preserved energy as well as isotropic vortex interaction. However, in our work the truncated wavelength was allowed to grow with time.

Numerical experiments were carried out to examine the effect of various minimum wavelengths. Each vortex was created at the edge of the splitter plate to satisfy the Kutta condition. A variable wavelength was assigned to each vortex blob as it was shed at the rear edge of the plate and its value increased with downstream distance. In this way, the instabilities found close to the splitter plate depended upon individual discrete vortices, and behaved in accord with the growth predicted by linear instability theory. However, downstream the instabilities were governed by the dynamics of the large-scale structure, since the length scales incorporated the effects of significant interactions such as vortex pairing.

A minimum starting cut-off of $2 \mathrm{~cm}$ yielded reasonable results for mean velocity, root mean square fluctuations and Reynolds stresses; these are presented in Figures 3, 4 and 5, respectively. In these calculations, the variable wavelength was always greater than the mean spacing of the large coherent structure. For example, after 300 time steps, the wavelength cut-off was $8 \mathrm{~cm}$.

It is of interest to note that a numerical experiment performed with a fixed cut-off of $8 \mathrm{~cm}$ yielded statistics identical to those of Figures 3-5. However, the computing time was significantly larger, since each vortex in the flow field had to participate in the interaction, yielding a computational time varying as $N^{2}$, where $N$ is the total number of vortices.

Although the quantitative results using the variable wavelengths and fixed wavelengths are quite similar to each other, the flow fields appeared very different. In particular, the roll-up of small scale instabilities yielded large elliptically shaded structures, similar to those photographed by Winant and Browand. ${ }^{3}$ The spiral arms, resembling nebulae in appearance, depicted the effect of vortex pairing in a fashion much more similar to experimental observations than the results of the fixed wavenumber calculation presented in Figure 1.

The variable cut-off is not a numerical artefact, since the statistical quantities measured are independent of this feature, yet it does change the visual appearance of the shear layer. An improved understanding of the mechanism of kinematic interactions between large and small vortices of various wavelengths should elucidate their effect in a tangible way.

\section{REFERENCFS}

1. G. Brown and A. Roshko, 'On density effects and large structure in turbulent mixing layers', J. Fluid Mech., 64, 775-816 (1974).

2. C. D. Winant and F. K. Browand, "Vortex pairing: the mechanisms of turbulent mixing layer growth at moderate reynolds number', $J$. Finid Mech., 63, 237-255 (1974).

3. A. J. Chorin, 'Numerical study of slightly viscous flow', J. Fluid Mech., 57, 785-796 (1973).

4. A. J. Chorin. 'Vortex sheet approximation of boundary layers', J. Comput. Physics, 27, $428-442$ (1978).

5. A. J. Chorin, 'Vortex methods and boundary layer instability', SIAM J. Sci. Stat. Comput., 1, 1-21 (1980).

6. B. Couet and A. Leonard, 'Mixing layer simulation by an improved three dimensional vortex-in-cell algorithm', Seventh International Conference on Numerical Methods in Fluid Dynamics, Stanford University, 23-27 June 1980.

7. L. Rosenhead, 'The formation of vortices from a surface discontinuity', Proc. Roy. Soc., A134, 170-192 (1932).

8. E. Acton, 'The modelling of large eddies in a two-dimensional shear layer', J. Fluid Mech., 76, 561-592 (1976).

9. H. Aref and E. D. Siggia, 'Vortex dynamics of the two-dimensional turbulent shear layer', J. Fluid Mech., 100, 705-737 (1980).

10. S. Lie and F. Engle, Theorie der Transformationsgruppen, Teubner, Liepzig, 1880.

11. O. H. Hald, 'Convergence of vortex methods for Euler's equations II', SI AM J. Numer. Anal., 16, (5), 726-755 (1979).

12. J. A. Laitone, 'A numerical solution for gas-particle flow at high Reynolds numbers,' J. Applied Mech., 48, 465-471 (1981).

13. A. Roshko, 'Structure of turbulent shear flows: a ncw look', AIAA J., 14, 1349-1357 (1976).

14. R. G. Batt, 'Turbulent mixing of passive and chemically reacting species in a low-speed shear layer', J. Fluid Mech., 82, 53-95 (1977)

15. W. T. Ashurst, 'Numerical simulation of turbulent mixing layers via vortex dynamics', in F. Durst (ed.), Turbulent Shear Flows, Springer-Verlag, 1979.

16. P. Bradshaw, 'The effects of initial conditions on the development of free shear layer', J. Fiuid Mech., 26, 225 (1966). 